\title{
CrystEngComm
}

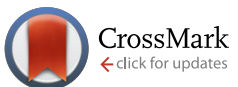

Cite this: CrystEngComm, 2016, 18 1918

Received 12th December 2015, Accepted 15th February 2016

DOI: $10.1039 / c 5 c e 02437 d$

www.rsc.org/crystengcomm

\section{A new nanocrystalline binary phase: synthesis and properties of cubic tin monoselenide $\dagger$}

\author{
Ran E. Abutbul, ${ }^{\text {ab }}$ Elad Segev, ${ }^{\text {b }}$ Shmuel Samuha, ${ }^{\text {ac }}$ Leila Zeiri, $^{\text {bd }}$ Vladimir Ezersky, $^{\text {b }}$ \\ Guy Makov ${ }^{\mathrm{ab}}$ and Yuval Golan*ab
}

\begin{abstract}
A new nanometric cubic binary phase of the tin mono-selenide system, $\pi$-SnSe, was obtained as cube shaped nanoparticles. Its structure and atomic positions were adopted from previously reported $\pi$-SnS $\left(P 2_{1} 3, a_{0}=11.7 \AA\right)$. The proposed structure model of $\pi$-SnSe, with 64 atoms per unit cell, was refined against experimental X-ray diffraction using Rietveld method ( $\left.a_{0}=11.9702(9) \AA ; R_{\mathrm{p}}=1.65 R_{\mathrm{wp}}=2.11\right)$. The optical properties of this new cubic SnSe phase were characterized by Raman and optical absorption spectroscopies. The optical band gap was assessed to be indirect, with $E_{\mathrm{g}}=1.28 \mathrm{eV}$ (in the near infrared), compared to $E_{\mathrm{g}}=0.9 \mathrm{eV}$ (indirect) and $1.3 \mathrm{eV}$ (direct) for the conventional orthorhombic phase of $\alpha-\mathrm{SnSe}$. Raman spectroscopy indicated significant phonon restraining, which is likely to be beneficial for thermoelectric applications. Since the new cubic phase belongs to a class of non-centrosymmetric crystals, interesting and potentially useful properties may arise. Density functional theory calculations have been applied in order to validate phase stability and evaluate the energy bandgap. These results, together with the recently discovered cubic phase of $\pi$-SnS, confirm the existence of a new class of nanoscale materials in the tin chalcogenide system.
\end{abstract}

\section{Introduction}

Considerable attention is being focused on narrow band gap materials because of their potential use in solar cells, optical communication and near-infrared (NIR) detectors. ${ }^{1,2}$ In particular, tin mono-sulfide (SnS) and tin mono-selenide (SnSe) which belong to the IV-VI semiconductor family have become attractive due to their promising opto-electronic properties, moderate toxicity, chemical stability and natural abundance. ${ }^{1}$ Conventional tin-chalcogenides adopt a layered orthorhombic structure (GeS type) with two smaller lattice constants and one much larger. Because of the resemblance in atomic positions, this structure could also be viewed as a distorted $\mathrm{NaCl}$ structure. Indeed, much effort has been directed toward improved syntheses of tin-chalcogenide nanomaterials. ${ }^{4-14}$

In the case of SnS, surfactant assisted synthesis of nanoparticles and chemical bath deposition (CBD) usually

\footnotetext{
${ }^{a}$ Department of Materials Engineering, Ben-Gurion University of the Negev, Beer-Sheva 84105, Israel. E-mail: ygolan@bgu.ac.il

${ }^{b}$ Ilse Katz Institute for Nanoscale Science and Technology, Ben-Gurion University of the Negev, Beer-Sheva 84105, Israel

${ }^{c}$ NRCN, P.O. Box 9001, Beer-Sheva 84190, Israel

${ }^{d}$ Chemistry Department, Ben-Gurion University of the Negev, Beer-Sheva 84105, Israel

$\dagger$ Electronic supplementary information (ESI) available: Methods and characterization techniques, Rietveld refinement data, crystallographic information file (CIF). See DOI: 10.1039/c5ce02437d
}

resulted in elongated platelet nanoparticles and flake like morphology respectively which corresponded to the orthorhombic structure of SnS referred to as $\alpha$-SnS. ${ }^{12-21}$ Yet, more recent publications reported on zinc blende (ZB) nanoparticles or thin films which formed under specific synthetic conditions. ${ }^{13,22-26}$ Transmission electron microscopy (TEM) of the materials was inconsistent with the lattice parameter expected for the ZB structure. In addition, density functional theory (DFT) calculations predicted this phase to be thermodynamically unstable. ${ }^{27}$ We have recently reported that the new phase of SnS in fact belongs to a new, simple cubic binary phase of SnS, which possesses an exceptionally large lattice parameter $\left(a_{0}=11.7 \AA\right)^{28}$ and which we designated as $\pi$-SnS. ${ }^{28}$ The equivalent orthorhombic analogue of $\alpha$-SnS, tin mono-selenide ( $\alpha$-SnSe), is an indirect band gap semiconducting material $(0.9 \mathrm{eV})$ with promising potential use in thermoelectric applications. ${ }^{7,10,29,30}$ In fact, the highest figure of merit for thermoelectric material efficiency has been reported for $\alpha$-SnSe at elevated temeperature. ${ }^{29} \mathrm{SnS}$ and SnSe share much in common, chemically and structurally. Hence, it was natural to question if the selenide analogue of the $\pi$-SnS prototype also existed in the form of $\pi$-SnSe.

In this publication, we report on a new cubic binary phase of tin mono-selenide, $\pi$-SnSe with a correspondingly large lattice parameter of $a_{0}=11.9702(9) \AA$ A. The described synthetic protocol enables synthesis of pure samples of $\alpha$-SnSe and $\pi$-SnSe upon demand, allowing for their characterization in 
the nanoscale as well as in the macroscale. The $\pi$-SnSe was characterized using an array of powerful techniques, including X-ray diffraction (XRD), Raman spectroscopy and optical absorption spectroscopy. The stability of the $\pi$-SnSe has been studied using DFT calculation along with evaluation of the material band gap.

\section{Experimental}

\section{(a) Materials}

Purified water $(18.2 \mathrm{M} \Omega \mathrm{cm})$ was obtained from Millipore filtration system. Tin(II) chloride $\left(\mathrm{SnCl}_{2}, 98 \%\right)$, oleylamine (OLA, $>98 \%$ ), oleic acid (OA, >90\%), 1-dodecanthiol (DDT, 98\%), selenourea (SU, >98\%), were all purchased from SigmaAldrich and used without further purification (unless stated otherwise in the text). Methanol (99.8\%) and chloroform (99.9\%) were purchased from Bio-Lab, Israel and used without further purification.

\section{(b) Synthesis of $\pi$-SnSe nanoparticles}

$5 \mathrm{ml}$ of OLA (15.53 mmol) held under vacuum overnight, $56.88 \mathrm{mg}$ of $\mathrm{SnCl}_{2}(0.3 \mathrm{mmol}) 100 \mu \mathrm{L}$ of water $(5.5 \mathrm{mmol})$ were placed in a $50 \mathrm{ml}$ three necked flask together with a magnetic stirrer bar. The flask was transferred to a heating mantle, evacuated and flushed with argon 4 times and stirred at $50{ }^{\circ} \mathrm{C}$ until a milky white suspension appeared. Then, 0.5 $\mathrm{ml}$ of OA was injected into the flask, which was then evacuated and left under vacuum for $30 \mathrm{~min}$. Simultaneously, $1 \mathrm{ml}$ of OLA (3.1 mmol) and $18.45 \mathrm{mg}$ of SU $(0.15 \mathrm{mmol})$ were placed in an amber vial together with a magnetic stirring bar. The vial was evacuated and flushed with argon 4 times and left under vacuum for $30 \mathrm{~min}$. Subsequently, the reaction flask was flushed with argon and the temperature was raised to $120{ }^{\circ} \mathrm{C}$ at a heating rate of 10 degrees per min. Once the reaction reached the destination temperature, the milky white colour disappeared to form a clear yellowish solution. The reaction was left for another $30 \mathrm{~min}$ while in the meantime the amber vial was transferred a bench-top sonicating bath to dissolve any residual aggregates of SU. The SU-OLA solution was injected to the reaction flask. The reaction turned reddish-brown within 10 seconds after injection. The incubation time was set to $5 \mathrm{~min}$ which after the heating mantle was removed. The reaction was allowed to cool naturally to $40{ }^{\circ} \mathrm{C}$ and $1 \mathrm{ml}$ of DDA was injected. The colloidal suspension was then stirred for another $5 \mathrm{~min}$ and $5 \mathrm{ml}$ of chloroform were added into the flask. The solution was then washed with a 1:10 ratio of chloroform:methanol and centrifuged at $2800 \mathrm{rpm}$ for $5 \mathrm{~min}$.

\section{(c) Synthesis of $\alpha$-SnSe nanoparticles}

$\alpha$-SnSe nanoparticles were synthesized similarly as the synthesis of the $\pi$-SnSe by keeping all reaction procedures and parameters identical, except for elevating the temperature of the reaction to $240{ }^{\circ} \mathrm{C}$ instead of $120{ }^{\circ} \mathrm{C}$. After injecting the
SU-OLA solution, the reaction solution instantly turned black.

\section{Results and discussion}

The two syntheses described above resulted in two different morphologies, thick platelets (Fig. 1a) and aggregated cubelike nanoparticles (Fig. 1b, e and f). The nanoparticles with thick platelet morphology exhibited wider size and shape distributions. The corresponding XRD pattern presented in Fig. 2a match to $\alpha$-SnSe (JCPDS no. 48-1224, $a_{0}=11.498 \AA$, $\left.b_{0}=4.153 \AA, c_{0}=4.440 \AA\right)$. The cube-like nanoparticles were subjected to EDS chemical analysis in TEM, and showed a $\mathrm{Sn}:$ Se ratio of $1: 1$ within the experimental error $(5 \%)$. Selected area electron diffraction (SAED) taken from a large cluster of nanoparticles yielded a ring pattern which could

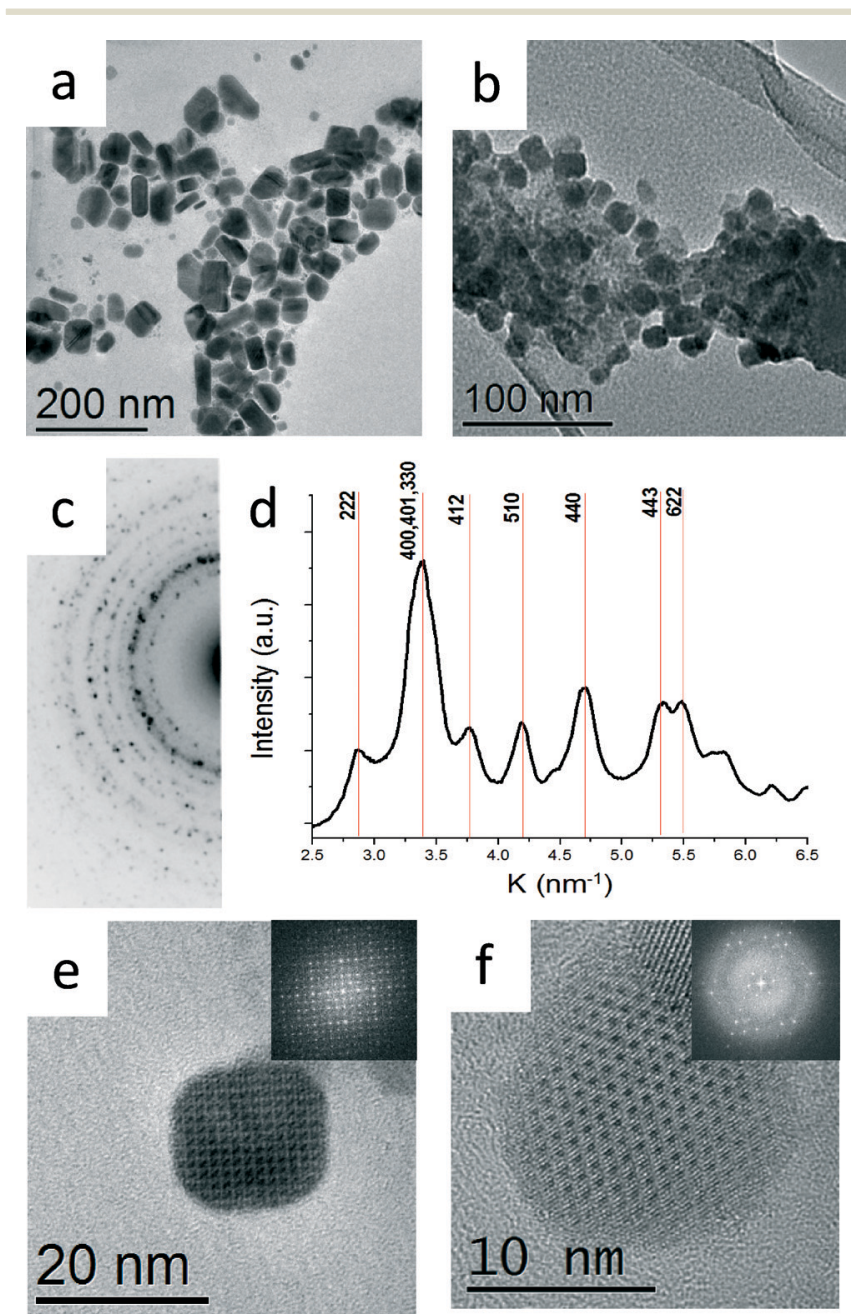

Fig. 1 Bright field TEM micrographs showing (a) $\alpha$-SnSe nanoparticles synthesized at $240{ }^{\circ} \mathrm{C}$ (b) $\pi$-SnSe nanoparticles synthesized at $120{ }^{\circ} \mathrm{C}$. (c) SAED taken from b. (d) Intensity profile deduced from the diffraction pattern shown in c obtained by radial integration of the diffraction ring intensities. ${ }^{3}$ (e) HRTEM micrograph showing a cube-like nanoparticle taken along [001] orientation. Inset: FFT of the HRTEM image (f) HRTEM micrograph showing a cube-like nanoparticle taken along [111] orientation. Inset: FFT of the HRTEM image. Both SAED and FFTs confirmed the proposed cubic structure with $a_{0}=12.1 \AA$. 

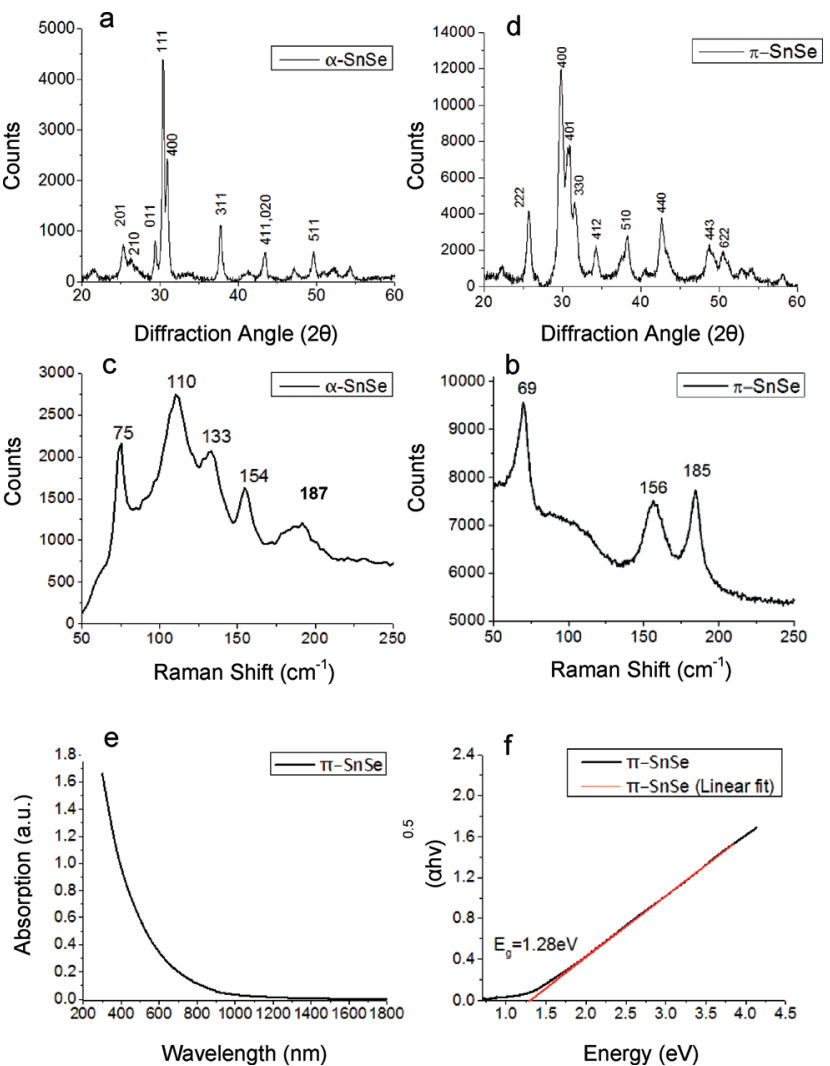

Fig. 2 Powder XRD patterns corresponding to (a) $\alpha$-SnSe with irregular morphologies (b) $\pi$-SnSe with cube-like morphology. Raman spectra corresponding to (c) $\alpha$-SnSe nanoparticles (d) $\pi$-SnSe nanoparticles. The new $\pi$-SnSe phase shows less features than conventional $\alpha$-SnSe in the investigated region. (e) UV-vis-NIR spectra of $\pi$-SnSe cube-like nanoparticles. (f) Tauc plot of the corresponding absorption spectra, indicating a band gap of $1.28 \mathrm{eV}$ for the $\pi$-SnSe phase.

not be indexed as $\alpha$-SnSe. A line profile was extracted from the SAED pattern by radial integration of the electron diffraction ring intensities in order to obtain the precise position of the reflections (Fig. 1c and d). ${ }^{3}$ The measured $d_{h k l}$-spacings acquired from the line plot differed substantially from the $d_{h k l}$-spacing expected for $\alpha$-SnSe. The XRD pattern acquired from the same sample corroborated these results (Fig. 2b).

High resolution TEM (HRTEM) micrographs of two cubelike nanoparticles at different zone axes (ZA) are presented in Fig. 1e and $\mathrm{f}$; the inset in Fig. 1e and $\mathrm{f}$ shows the corresponding fast Fourier transform (FFT) pattern. The FFT patterns are in agreement with the $d_{h k l}$-spacing extracted from SAED patterns. From the analysis of the net symmetry presented in these FFT patterns, it was plausible to assume that the $\pi$-SnSe crystallized in a cubic symmetry with $a_{0}=$ $12.1 \pm 0.04 \AA$.

In a recent report from our group, we have used the precession electron diffraction tomography (PEDT) technique for obtaining a full structure solution for the $\pi$-SnS phase $\left(P 2_{1} 3\right.$, $a_{0}=11.7 \AA$ ), a previously unknown simple cubic binary phase of tin mono-sulfide. ${ }^{28}$ Significant progress in the synthesis allowed us to obtain pure powder samples of the $\pi$-SnS nanocrystalline phase. Subsequently, refinement of the structural model was carried out using Rietveld refinement on powder $\mathrm{X}$-ray diffraction data, establishing a refined lattice parameter of $a_{0}=11.595 \AA^{31}$ Moreover, the model also confirms with solution deposited thin film samples of $\pi$-SnS. ${ }^{32,33}$ Assuming that the tin mono-selenide phase belong to the $\pi$-SnS structural type, both electron diffraction data and XRD could be indexed appropriately. By replacing $\mathrm{S}$ with Se atom type, a theoretical $\pi$-SnSe structural model was proposed. Then, applying the Rietveld method utilized in FULLPROF software, ${ }^{34}$ the theoretical $\pi$-SnSe model was fitted to the experimental powder XRD data. Negligible difference between calculated and observed X-ray diffraction profiles along with low values of reliability factors $\left(R_{\mathrm{p}}=1.65 R_{\mathrm{wp}}=2.11\right)$, obtained at the final Rietveld $\pi$-SnSe structure refinement, confirmed the applicability of the structural solution of the $\pi$-SnS for the $\pi$-SnSe phase. The results are presented in detail in Fig. S1 in ESI. $\dagger$ The refined lattice parameter of $\pi$-SnSe was determined to be $a_{0}=11.9702(9) \AA$. XRD and electron diffraction in TEM are different techniques for lattice parameter determination. Thus, deviation in the results can be expected $\left(a_{0}=\right.$ 11.9702(9) $\AA$ in XRD vs. $a_{0}=12.1$ in TEM $\AA$, a $1.07 \%$ difference). Yet, excellent match has been achieved by different techniques. We note that XRD is the most accurate technique for determination of the lattice parameter of powder samples.

Comparison of the relative atomic positions of $\pi$-SnSe with those of $\pi$-SnS shows that they do not differ from one other by more than a few percent, with the lattice parameters scaling similarly to within less than $3.2 \%$. These variations are well expected since the selenide anion is larger than the sulfide anion. As discussed above, $\mathrm{SnS}$ and SnSe are part of the IV-VI family of semiconductor materials, and share similarities with GeS and GeSe, namely belong to the orthorhombic GeS prototype. The discovery of $\pi$-SnS and $\pi$-SnSe could point toward a new simple cubic prototype in this system, yet no similar simple cubic phase for GeS or GeS has been reported to date. We also note that the new cubic phase, $\pi$-SnSe belongs to a class of non-centrosymmetric crystals. This, in turn, gives rise to piezoelectricity, optical activity and enantiomorphism - an important and potentially useful material properties dictated by crystal symmetry. While this warrants further consideration, both experimental and theoretical, we note that these features are not expected for the orthorhombic crystal structure.

Since our synthetic protocol allows synthesizing each $\mathrm{SnSe}$ phase separately, we have explored the properties of $\pi$-SnSe using macroscopic characterization tools. Fig. 2 shows the Raman spectra of both $\pi$-SnSe and $\alpha$-SnSe. The $\alpha$-SnSe spectrum shows peaks at $75 \mathrm{~cm}^{-1}, 110 \mathrm{~cm}^{-1}, 133 \mathrm{~cm}^{-1}, 154 \mathrm{~cm}^{-1}$, $187 \mathrm{~cm}^{-1}$. The $110 \mathrm{~cm}^{-1}$ peak corresponds to the $B_{3 g}$ mode, while the peaks at $75 \mathrm{~cm}^{-1}, 133 \mathrm{~cm}^{-1}, 154 \mathrm{~cm}^{-1}$ belong to $A_{g}$ phonon modes, all attributed to $\alpha$-SnSe. ${ }^{7,35-37}$ The peak at $185 \mathrm{~cm}^{-1}$ belongs to $\mathrm{SnSe}_{2} \cdot{ }^{38}$ It was found that both $\alpha$-SnSe and $\pi$-SnSe particles are sensitive to the excitation laser beam, which causes them to transform from $\mathrm{SnSe}$ to $\mathrm{SnSe}_{2}$ over time. The Raman spectrum of $\pi$-SnSe shows only 3 sharp 
peaks in the same region, $69 \mathrm{~cm}^{-1}, 156 \mathrm{~cm}^{-1}$ and $185 \mathrm{~cm}^{-1}$. Another weak and broad peak is centered around $120 \mathrm{~cm}^{-1}$. The peak at $185 \mathrm{~cm}^{-1}$ is again attributed to $\mathrm{SnSe}_{2}$ while the other peaks are assigned to $\pi$-SnSe. Comparing the two spectra shows that the $\pi$-SnSe $110 \mathrm{~cm}^{-1}$ and $133 \mathrm{~cm}^{-1}$ peaks are substantially weakened. Such changes are expected due to the different structure, and the subsequent lattice symmetry of the two phases. The $185 \mathrm{~cm}^{-1}$ peak is sharper in the spectrum of $\pi$-SnSe. We speculate that the larger surface area in $\pi$-SnSe nanoparticles makes them more sensitive to the laser beam, causing them to readily transform from $\pi$-SnSe to $\mathrm{SnSe}_{2}$. Surprisingly, comparison of the Raman spectra of $\alpha$-SnS and $\pi$-SnS showed quite a different picture. While $\pi$-SnS showed several additional peaks compared to $\alpha$-SnS, ${ }^{31}$ Fig. 2d shows restraining of phonons in the Raman spectrum of the $\pi$-SnSe phase as compared $v s$. $\alpha$-SnSe. This phenomenon of phonon restraining could be related to the change in the tin and selenide coordination. It is well known that upon heating the orthorhombic phase of SnSe, a second order phase transition occurs from Pnma (space group no. 62) to $\mathrm{Cmcm}$ (space group no. 63). This transition could be described as a change in the coordination polyhedron of the tin and selenide ions, with higher symmetry in the high temperature orthorhombic phase. The higher site symmetry results in phonon anharmonicity and substantial phonon scattering. ${ }^{39}$ Examination of the discussed cubic crystal structure with respect to the low temperature orthorhombic structure suggests that a similar transition may be occurring. In this case, both tin and selenide ions are located in " $4 a$ " Wyckoff sites (space group no. 198) with each ion surrounded by three ions of the opposite valence in 3-fold symmetry. Thus, the coordination polyhedron for both tin and selenium ions could be represented as a pyramidal sub-structure in the $\pi$-SnSe crystal. We speculate that this unique arrangement could adversely affect phonon propagation in the material, similarly to the previously proposed mechanism. ${ }^{39}$ This mechanism of phonon anharmonicity has been reported to be responsible for the ultra-low thermal conductivity of $\alpha$-SnSe at high temperatures. ${ }^{29,39}$ Thus, we conclude that while substantial phonon scattering is observed in orthorhombic $\alpha$-SnSe only at high temperature, the new cubic $\pi$-SnSe is expected to exhibit significant phonon scattering also at room temperature. Further studies are underway in order to explore these phenomena and to assign the specific phonon modes to the peaks in the Raman spectrum of $\pi$-SnSe.

The optical properties of $\pi$-SnSe were explored using UVvis-NIR absorption spectroscopy. The $\pi$-SnSe absorption curves and the corresponding Tauc plot for $\pi$-SnSe cube-like nanoparticles are shown in Fig. 2. We note that the literature reported band of $\alpha$-SnSe is $0.9 \mathrm{eV}$ (indirect) and $1.3 \mathrm{eV}$ (direct). ${ }^{6,7,9,10,30,37}$ The absorption curve in Fig. 2e shows an onset of absorption around $\sim 950 \mathrm{~nm}$. The band gap of an indirect bandgap semiconductor is related to the absorption coefficient $\alpha$ according to the formula $\alpha h v^{0.5}=A\left(h v-E_{\mathrm{g}}\right)$ where $h$ is Planck's constant, $v$ is the light frequency, $E_{\mathrm{g}}$ is the material band gap and $A$ is a constant. The band gap can be obtained from the $x$-intercept upon plotting $(\alpha h v)^{0.5} v s$. $h v$, as shown in Fig. 2f. The plot yielded a straight line, indicating that $\pi$-SnSe is an indirect band gap material, with the extrapolated fitted line intercepting the $h v$ axis at $1.28 \mathrm{eV}$. Thus, the bandgap of $\pi$-SnSe is significantly larger than that of $\alpha$-SnSe, with a difference of about $0.4 \mathrm{eV}$. This implies that while $\alpha$-SnSe is active in the short wave infrared range, the new $\pi$-SnSe phase is expected to be active closer to the visible range, with technological implications for solar cells, thermoelectric applications and photocatalysis. ${ }^{1,2,40,41}$ A similar increase was reported also for $\pi$-SnS with respect to $\alpha$-SnS, where the band gap is increased from $1.07 \mathrm{eV}$ in conventional $\alpha$-SnS to $1.53-1.72 \mathrm{eV}$ in $\pi$-SnS. ${ }^{31-33}$

The stable phase of tin selenide in the solid state is the 8 atom orthorhombic $\alpha$-SnSe with the space group Pnma. We performed DFT-PBE calculations of this orthorhombic 8 atom phase and of the rocksalt phase for reference purposes. For the orthorhombic phase, the calculated lattice parameters were found to be $a_{0}=11.8346 \AA, b_{0}=4.2254 \AA, c_{0}=4.54 \AA$, compared to the experimental values of JCPDS no. 48-1224, $a_{0}=11.498 \AA, b_{0}=4.153 \AA, c_{0}=4.440 \AA$. Representing deviations of up to several percent as might be expected. The electronic band structure in the relaxed position was obtained and is presented in Fig. 3a.

It can be seen that an indirect band-gap of $c a .0 .597 \mathrm{eV}$ is found between the points $\mathrm{Z}$ and $\mathrm{X}$ and a direct band-gap of ca. $0.867 \mathrm{eV}$ is found between the high symmetry points $\mathrm{Z}$ and $\mathrm{G}$, in excellent, but probably fortuitous, agreement with experimental measurements of $0.9 \mathrm{eV} .^{7,9,10,30,37}$

To study the 64 atom $\pi$-SnSe phase we constructed a simple cubic unit cell consisting of the 64 atoms initially located in their experimentally determined positions. A structural relaxation allowing the atomic positions, the volume and the lattice constants of the unit cell to vary was then performed. In this cell, convergence of the calculation was achieved with a $8 \times 8 \times 8 k$-point mesh. A total energy of -829.8196 Ry per 64 atom unit cell, a total (isotropic) stress of $-0.32 \mathrm{kbar}$ and a relaxed lattice parameter of 12.284 which is slightly larger than the experimental value of 11.9702(9) A reported above indicating that the phase is mechanically stable. The stability of the $\pi$-SnSe phase can be examined by comparing the total energy per atom pair of -25.9319 Ry to $-25.9342 \mathrm{Ry}$ in the ideal rocksalt phase and -25.9339 Ry in the orthorhombic phase. We see that the total energy results predict the $\pi$-SnSe phase to be less stable than the orthorhombic phase by approximately $2 \times 10^{-3} \mathrm{Ry}$ per atom which is computationally significant and at the limit of physical significance. The orthorhombic $\alpha$-SnSe phase is slightly less stable in our calculation than the energetically equivalent rocksalt phase, but this difference is physically insignificant and of the same order of magnitude as found in earlier studies. ${ }^{42}$

To examine the origin of the stability of the $\pi$-SnSe phase relative to the ideal rocksalt structure we examined the band structure of both systems in a unit cell containing 64 atoms, i.e. with a similar size of the Brillouin zone. The results are 
a

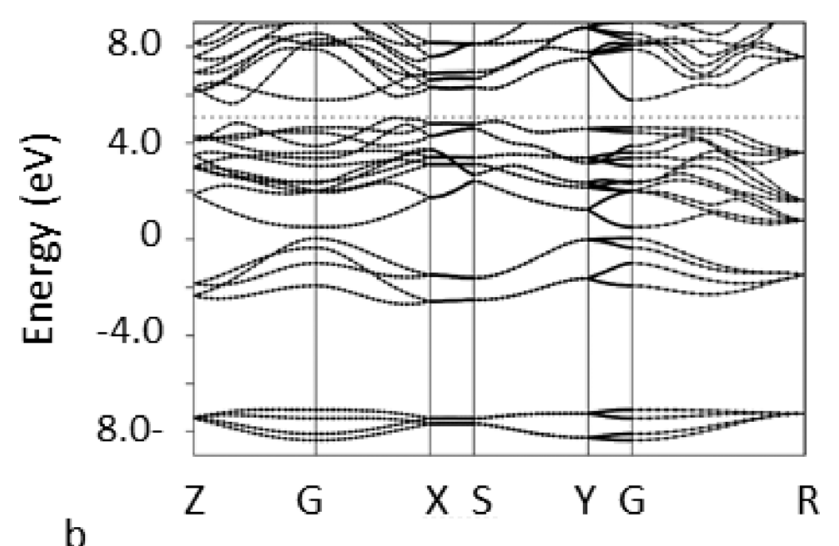

b
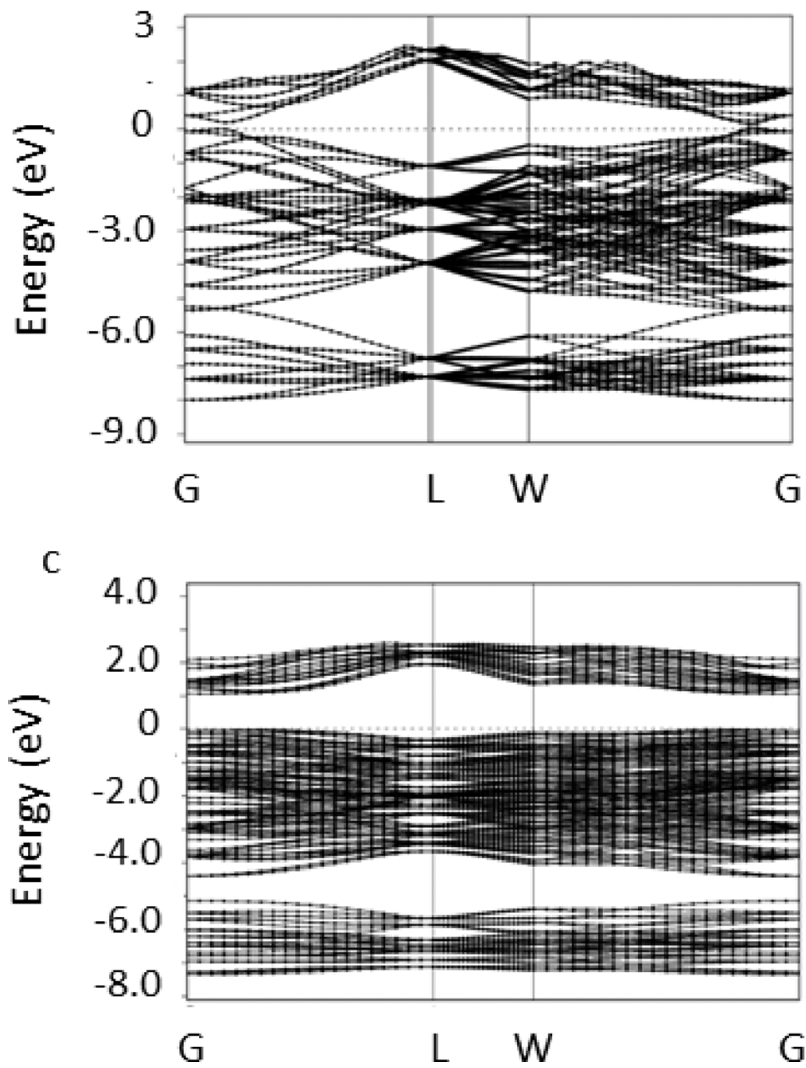

Fig. 3 (a) Band structure of the orthorhombic $\alpha$-SnSe phase, with 8 atoms in the Herzenbergite structure. (b) Calculated band structures of the ideal rock salt lattice with 64 atoms per unit cell (c) and the $\pi$-SnSe phase with 64 atoms per unit cell.

shown in Fig. 3c. It is immediately seen that the band structure in the $\pi$-SnSe phase is distorted relative to the highly symmetric structure in the ideal rocksalt phase, as can be seen, e.g., at the L point. More significantly, the energy gap between the valence and conduction band is found to increase from $c a .0 .2 \mathrm{eV}$ in the ideal structure to approximately $1.18 \mathrm{eV}$ in the $\pi$-SnSe phase in very good agreement with the measurements reported above $(1.28 \mathrm{eV})$. We note that DFTPBE is well-known to underestimate band gaps. ${ }^{43}$ Due to the large size of the unit cell, and the consequently small size of the Brillouin zone, it was impossible to determine from our calculations whether the band gap is indirect.

\section{Conclusions}

We have reported on a new binary phase of tin mono-selenide, $\pi$-SnSe. A proposed theoretical $\pi$-SnSe model, based on the recently discovered $\pi$-SnS structure was constructed by replacing $\mathrm{S}$ ion with Se ions. Using this modified model, we successfully indexed both XRD and electron diffraction patterns obtained for the new $\pi$-SnSe material. Rietveld refinement of the modified $\pi$-SnSe model was carried on XRD data. The high compatibility between the modified $\pi$-SnSe model and observed XRD profiles, along with low reliability factors, provided strong proof for the correctness of the $\pi$-SnSe model, and points towards a new prototype which may exist in parallel to the GeS prototype. Since the $\pi$-SnSe phase was obtained as a single phase of pure cube-like nanoparticle powder, it was possible to carry out Raman spectroscopy and UV-vis-NIR absorption spectroscopy. Raman spectroscopy showed a distinct fingerprint for $\pi$-SnSe, with restrained phonons (smaller number of peaks) in comparison to $\alpha$-SnSe. This phenomenon could be potentially useful for thermoelectric applications where low thermal conductivity is desired. UV-vis-NIR spectroscopy indicated an indirect band gap of $c a .1 .28 \mathrm{eV}$, which is larger by about $0.4 \mathrm{eV}$ with respect to that of $\alpha$-SnSe $(0.9 \mathrm{eV}$, indirect). Due to the different crystallographic structure, this new cubic phase of SnSe is predicted to exhibit interesting and potentially useful piezoelectric properties, unlike the conventional orthorhombic phase. DFT total energy calculations indicated that the $\pi$-SnSe is mechanically stable and is energetically close to the stable $\alpha$-SnSe phase. The band gap is confirmed to increase from $c a .0 .9$ to $1.2 \mathrm{eV}$ in very good, but possibly fortuitous, agreement with the experimental measurements.

\section{Acknowledgements}

We thank Dr. Dimitry Mogilyanski for expert assistance with XRD, Dr. Iddo Pinkas for expert assistance with Raman spectroscopy and Eyal Toutian for assistance with nanoparticle synthesis. This work was supported by the Israel Science Foundation under Grant \# 340/2010, and by the Focal Technological Area (FTA) Program of the Israel National Nanotechnology Initiative (INNI).

\section{References}

1 L. M. Peter, Philos. Trans. R. Soc., A, 2011, 369, 1840-1856.

2 S. Abermann, Sol. Energy, 2013, 94, 37-70.

3 C. Gammer, C. Mangler, C. Rentenberger and H. P. Karnthaler, Scr. Mater., 2010, 63, 312-315.

4 B. K. Patra, S. Sarkar, A. K. Guria and N. Pradhan, J. Phys. Chem. Lett., 2013, 4, 3929-3934.

5 J. Ning, K. Men, G. Xiao, L. Wang, Q. Dai, B. Zou, B. Liu and G. Zou, Nanoscale, 2010, 2, 1699-1703.

6 J. J. Ning, G. J. Xiao, T. Jiang, L. Wang, Q. Q. Dai, B. Zou, B. B. Liu, Y. J. Wei, G. Chen and G. T. Zou, CrystEngComm, 2011, 13, 4161-4166. 
7 S. L. Zhao, H. A. Wang, Y. Zhou, L. Liao, Y. Jiang, X. Yang, G. C. Chen, M. Lin, Y. Wang, H. L. Peng and Z. F. Liu, Nano Res., 2015, 8, 288-295.

8 S. G. Hickey, C. Waurisch, B. Rellinghaus and A. Eychmuller, J. Am. Chem. Soc., 2008, 130, 14978-14980.

9 L. Li, Z. Chen, Y. Hu, X. Wang, T. Zhang, W. Chen and Q. Wang, J. Am. Chem. Soc., 2013, 135, 1213-1216.

10 D. D. Vaughn 2nd, S. I. In and R. E. Schaak, ACS Nano, 2011, 5, 8852-8860.

11 P. Ramasamy, P. Manivasakan and J. Kim, CrystEngComm, 2015, 17, 807-813.

12 X. Liu, Y. Li, B. Zhou, X. Wang, A. N. Cartwright and M. T. Swihart, Chem. Mater., 2014, 26, 3515-3521.

13 Z. Deng, D. Cao, J. He, S. Lin, S. M. Lindsay and Y. Liu, ACS Nano, 2012, 6, 6197-6207.

14 A. J. Biacchi, D. D. Vaughn 2nd and R. E. Schaak, J. Am. Chem. Soc., 2013, 135, 11634-11644.

15 A. de Kergommeaux, M. Lopez-Haro, S. Pouget, J. M. Zuo, C. Lebrun, F. Chandezon, D. Aldakov and P. Reiss, J. Am. Chem. Soc., 2015, 137, 9943-9952.

16 K. Ramasamy, V. L. Kuznetsov, K. Gopal, M. A. Malik, J. Raftery, P. P. Edwards and P. O'Brien, Chem. Mater., 2013, 25, 266-276.

17 G. H. Yue, Y. D. Lin, X. Wen, L. S. Wang and D. L. Peng, J. Mater. Chem., 2012, 22, 16437-16441.

18 D. D. Vaughn 2nd, O. D. Hentz, S. Chen, D. Wang and R. E. Schaak, Chem. Commun., 2012, 48, 5608-5610.

19 L. L. Cheng, M. H. Liu, M. X. Wang, S. C. Wang, G. D. Wang, Q. Y. Zhou and Z. Q. Chen, J. Alloys Compd., 2012, 545, 122-129.

20 W. Cai, J. Hu, Y. S. Zhao, H. L. Yang, J. Wang and W. D. Xiang, Adv. Powder Technol., 2012, 23, 850-854.

21 Y. Zhang, J. Lu, S. Shen, H. Xu and Q. Wang, Chem. Commun., 2011, 47, 5226-5228.

22 E. C. Greyson, J. E. Barton and T. W. Odom, Small, 2006, 2, 368-371.

23 D. Avellaneda, M. T. S. Nair and P. K. Nair, J. Electrochem. Soc., 2008, 155, D517-D525.

24 A. Akkari, C. Guasch and N. Kamoun-Turki, J. Alloys Compd., 2010, 490, 180-183.
25 Z. Deng, D. Han and Y. Liu, Nanoscale, 2011, 3, 4346-4351.

26 I. Y. Ahmet, M. S. Hill, A. L. Johnson and L. M. Peter, Chem. Mater., 2015, 27, 7680-7688.

27 L. A. Burton and A. Walsh, J. Phys. Chem. C, 2012, 116, 24262-24267.

28 A. Rabkin, S. Samuha, R. E. Abutbul, V. Ezersky, L. Meshi and Y. Golan, Nano Lett., 2015, 15, 2174-2179.

29 L. D. Zhao, S. H. Lo, Y. Zhang, H. Sun, G. Tan, C. Uher, C. Wolverton, V. P. Dravid and M. G. Kanatzidis, Nature, 2014, 508, 373-377.

30 W. J. Baumgardner, J. J. Choi, Y. F. Lim and T. Hanrath, J. Am. Chem. Soc., 2010, 132, 9519-9521.

31 R. E. Abutbul, E. Segev, L. Zeiri, V. Ezersky, G. Makov and Y. Golan, RSC Adv., 2016, 6, 5848-5855.

32 P. K. Nair, A. R. Garcia-Angelmo and M. T. S. Nair, Phys. Status Solidi A, 2016, 213, 170-177.

33 A. R. Garcia-Angelmo, R. Romano-Trujillo, J. CamposÁlvarez, O. Gomez-Daza, M. T. S. Nair and P. K. Nair, Phys. Status Solidi A, 2015, 212, 2332-2340.

34 J. Rodrigues-Carvajal, Program FULLPROF-98, Version 5.06, 2015.

35 H. R. Chandrasekhar, R. G. Humphreys, U. Zwick and M. Cardona, Phys. Rev. B: Solid State, 1977, 15, 2177-2183.

36 M. Parenteau and C. Carlone, Phys. Rev. B: Condens. Matter Mater. Phys., 1990, 4, 5227-5234.

37 J. Zhang, H. Y. Zhu, X. X. Wu, H. Cui, D. M. Li, J. R. Jiang, C. X. Gao, Q. S. Wang and Q. L. Cui, Nanoscale, 2015, 7, 10807-10816.

38 D. G. Mead and J. C. Irwin, Solid State Commun., 1976, 20, 885-887.

39 C. W. Li, J. Hong, A. F. May, D. Bansal, S. Chi, T. Hong, G. Ehlers and O. Delaire, Nat. Phys., 2015, 11, 1063-1069.

40 Z. Zhang, W. Wang, L. Wang and S. Sun, ACS Appl. Mater. Interfaces, 2012, 4, 593-597.

41 S. B. Rawal, S. Bera, D. Lee, D. J. Jang and W. I. Lee, Catal. Sci. Technol., 2013, 3, 1822-1830.

42 A. Walsh and G. W. Watson, J. Phys. Chem. B, 2005, 109, 18868-18875.

43 Y. Zhao and D. G. Truhlar, J. Chem. Phys., 2009, 130, 074103. 